J. clin. Path., 33, Suppl. (Ass. Clin. Path.), 8, 26-30

\title{
The measurement of cholecystokinin
}

\author{
JENS F. REHFELD \\ From the Institute of Medical Biochemistry, University of Aarhus, DK-8000 Aarhus C, Denmark
}

Measurement of hormones in biological fluids is an obvious prerequisite for evaluation of their physiological and pathophysiological significance. Of the steadily increasing list of gastrointestinal hormones, cholecystokinin (CCK) was the third to be discovered (Ivy and Oldberg, 1928). Cholecystokinin, secretin, and gastrin constitute the classical triad of gut hormones which, until recently, was assumed to exert the entire humoral control of digestion (Grossman, 1970a).

While it is now possible to measure the plasma concentration of gastrin, secretin, and most of the other chemically defined hormonal peptides from the gut (reviewed by Rehfeld, 1978a), all attempts to measure CCK in plasma have, in the author's opinion, failed.

An interest in the molecular nature of gastrin and gastrin-like peptides, and experience of assays for a number of gastrointestinal and other hormones, led me to establish the radioimmunoassay of CCK. After initial difficulties with the labelling of CCK had been overcome, sensitive and sequence-specific radioimmunoassays for CCK were developed (Rehfeld, 1978b). However, for several reasons, these assays proved unsatisfactory when applied to plasma. As foreseen several years ago using crossreacting gastrin assays (unpublished data), CCK occurs in several forms and their concentration in plasma is very low. Moreover, plasma proteins interfere greatly in the assay, and some plasma enzymes probably degrade CCK. These problems still have to be overcome. Nevertheless, it is hoped that the following account may be of some value.

\section{Development of the cholecystokinin concept}

Claude Bernard (1856) noted that when hydrochloric acid was introduced into the duodenum, the flow of bile increased. This occurred even after the vagus and the thoracic sympathetic nerves had been cut (Wertheimer, 1903). Fleig suggested in 1903, on the basis of experiments on dogs, that a humoral substance from the gut stimulated bile secretion and flow, and in 1904 he postulated the existence of a specific 'sapocrinin' released by soaps. Proof of the existence of a specific humoral substance which stimulates gall bladder contraction was finally obtained by Ivy and Oldberg (1928) and they named it cholecystokinin instead of sapocrinin. Subsequently, Ivy et al. (1930) and Sandblom (1933) showed the same mechanism in man. More recently Jorpes and Mutt (1966) showed that pancreozymin in the gut was identical to cholecystokinin and, consequently, it was proposed by Grossman (1970b) that the peptide should be termed only cholecystokinin (or CCK), instead of the cumbersome cholecystokinin-pancreozymin (or CCK PZ). This proposal is now generally accepted.

\section{Structure of a cholecystokinin isolated from} porcine gut

A major step towards the understanding of the nature of CCK occurred when Mutt and Jorpes (1967, 1968, 1971) purified and determined the structure of a triacontatriapeptide (CCK 33) isolated from the upper small intestine of hogs. Elucidation of the amino-acid sequence of CCK 33 (Fig. 1) disclosed some fascinating features. (1) The $\mathrm{COOH}$ terminal pentapeptide amide is identical to the $\mathrm{COOH}$ terminus of gastrin and to that of the frog peptide caerulein. ${ }^{1}$ (2) An O-methyl-sulphated tyrosyl is located in the same position as in caerulein ( 7 from the $\mathrm{COOH}$ terminus); it is shifted only one $¥$ position compared with gastrin. (3) The biological 웅 activity of CCK 33 is localised to the $\mathrm{COOH} \rightarrow$ terminus as in gastrin, and the potency is dependent on sulphation of the tyrosyl residue. A few years ago $\bar{N}$ Mutt (1976) reported that the gut extracts contained, $\sigma$ in addition to CCK 33, another CCK of similar $N$ activity with an additional six amino-acid residues $\underset{\omega}{N}$ linked at the $\mathrm{NH}_{2}$ terminus.

The structure of CCK has been confirmed in part

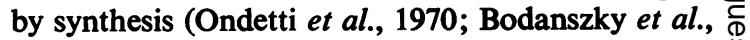
1972). During investigations of the contractile effect $\stackrel{\mathscr{\rho}}{\rightarrow}$ on the gall bladder of synthetic $\mathrm{COOH}$ terminal 0 fragments, Rubin and Engel (1973) unexpectedly discovered that some of the small fragments were

1 Caerulein is a peptide first isolated from the skin of Litoria caerulea and found to cause potent contraction of the gall bladder and secretion of pancreatic enzymes in mammals. 


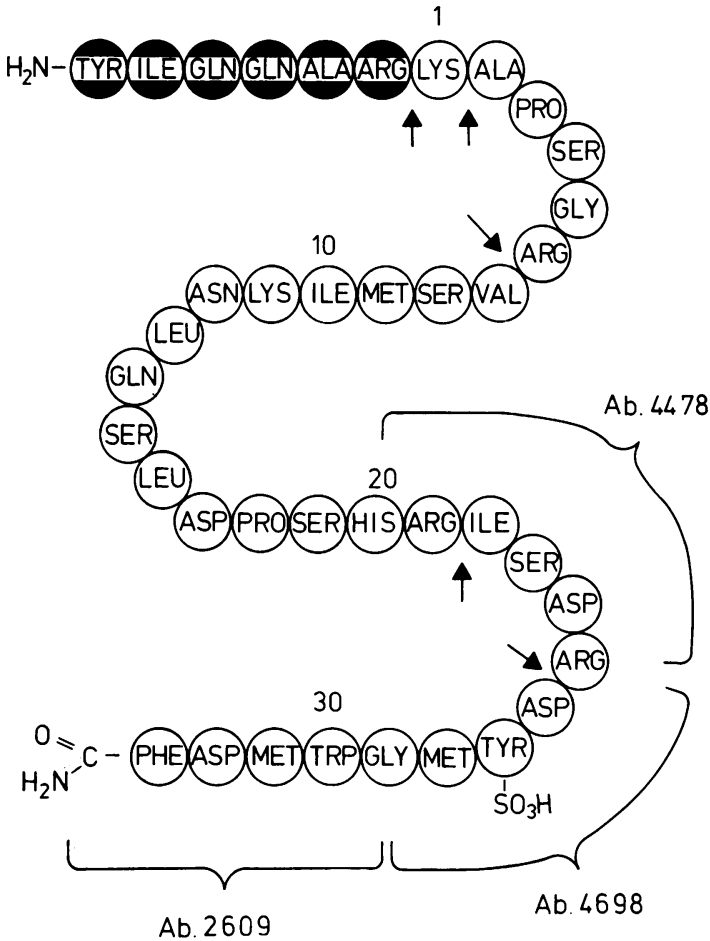

Fig. 1 Amino-acid sequence of porcine cholecystokinin 39 and cholecystokinin 33 (open circles). Brackets indicate sequence specificy of antisera developed by the author. Arrows indicate pcints of tryptic cleavage.

more potent than CCK 33. This important observation suggested that the principal molecular form of CCK might be a smaller $\mathrm{COOH}$ terminal fragment of CCK 33. The octapeptide (CCK 8) proved to be three times more active than CCK 33. As shown below, there are now good reasons for regarding CCK 8 as the principal molecular form instead of CCK 33.

\section{Method of assay}

\section{BIOASSAY VERSUS RADIOIMMUNOASSAY}

Originally CCK could be assayed only by biological methods. Radioimmunoassay is now possible and has the general advantages of low detection limits, necessary for measurements in blood, a high degree of specificity, good reproducibility relative to bioassay, and greater convenience. Bioassays have the disadvantage of measuring the resultant activity of any unrelated substances in the samples which affect the preparation used, whether they possess stimulatory or inhibitory activity. In contrast, immunoassays depend on the structure of a com- pound and measure a specific sequence of 4 to 6 amino-acids. Hence, bio- and immunoassays are complementary rather than alternatives.

PREPARATION OF ${ }^{125}$ I-CCK

As discussed in detail by Hunter (1971) ${ }^{125} I$ is, practically speaking, the only useful isotope for radioimmunoassays of peptide hormones. Polypeptide hormones have almost exclusively been iodinated at their tyrosyl residues by oxidation procedures using chloramine $\mathrm{T}$ or hydrogen peroxide/lactoperoxidase. CCK 33 contains one tyrosyl residue only, but this is sulphated and consequently is difficult to iodinate. This problem can be circumvented either by iodination of CCK 39, which contains an unsulphated $\mathrm{NH}_{2}$ terminal tyrosyl, or by iodination of desulphated CCKs. However, these oxidation procedures are deleterious to CCK because they oxidise the three methionyl residues (positions 9, 28, and 31 of CCK 33) which destroys both bioreactivity (Mutt, 1964) and immunoreactivity (Rehfeld, 1978b) virtually irreversibly. Consequently, CCK must be labelled by nonoxidative methods irrespective of which form of CCK is used.

The Bolton-Hunter reagent ( ${ }^{25} \mathrm{I}$-hydroxyphenylpropionic-acid-succinimide ester) has proved to be satisfactory; it presumably couples to the $\mathrm{NH}_{2}$ terminal lysyl residue of CCK 33. Its uptake is consistently 30 to $35 \%$ and the specific activity is $0.5 \mathrm{mCi} / \mathrm{nmol} \mathrm{CCK}$. The immunoreactivity of the labelled CCK is indistinguishable from that of native CCK, and the labelled compound can be used for more than 3 months (Rehfeld, 1978b).

\section{PRODUCTION OF CCK ANTISERA}

Both guinea pigs and rabbits produced good antisera when immunised with $20 \%$ pure porcine CCK; the CCK was coupled covalently to bovine serum albumin for immunising rabbits, but was given uncoupled in Freund's adjuvant to the guinea pigs (Rehfeld, 1978b). All the CCK-immunised animals in our laboratory produced antisera which gave good standard curves when the labelled CCK was prepared with the Bolton-Hunter reagent. The uncoupled porcine CCK is strongly antigenic in guinea pigs, suggesting that the structure of guinea pig CCK differs substantially from that of porcine CCK 33. Therefore, the guinea pig is recommended for antibody production.

OTHER REAGENTS IN CCK RADIOIMMUNOASSAY Plasma-coated charcoal has proved a simple and uncomplicated separation reagent. As standards we have used $99 \%$ pure porcine CCK 33 or CCK 8 . 
VALIDATION OF THE CCK RADIOIMMUNOASSAY As stated above, the $\mathrm{COOH}$ terminal pentapeptide sequence of CCK is identical to that of gastrin. Since this sequence is highly antigenic many antisera raised against CCK also react with gastrin. By immunising a large number of animals with cheap, impure antigen, however, a number of antisera specific for sequences 20 to 25 and 25 to 30 of CCK 33 were also obtained (Rehfeld, 1978b). This battery of sequence-specific antisera has proved indispensable for the characterisation of CCK and gastrin in the nervous system and cerebrospinal fluid (Uvnäs-Wallensten et al., 1977; Rehfeld, 1978c, d; Rehfeld and Kruse-Larsen, 1978; Larsson and Rehfeld, 1979), for phylogenetic studies (Larsson and Rehfeld, 1977a, b), and for evaluation of the quality of synthetic analogues (U. Ragnarsson and J. F. Rehfeld, unpublished data).

Antisera with different sequence specificities may also be produced by immunisation with pure fragments. Until recently, however, such fragments were very expensive or unobtainable.

\section{The measurement of cholecystokinin in tissue extracts}

The apparent concentration and molecular heterogeneity of CCK in tissue depend on the extraction method employed (Dockray, 1977; Muller et al., 1977; Rehfeld, 1978c). Thus, predominantly small molecular forms are released by the use of boiling water at neutral $\mathrm{pH}$, whereas larger forms are released at low $\mathrm{pH}$.

\section{THE GASTROINTESTINAL TRACT}

The concentration of specific immunoreactive $\mathrm{CCK}$ throughout the gut is shown in Table 1. Most of the intestinal CCK is located in the duodenum and jejunum. The stomach contains none, but the ileum and the colon contain small amounts, located

\begin{tabular}{lll}
\hline & $C C K 8$ & $C C K$ 33-39 \\
\hline Antrum & $<0 \cdot 2$ & $<0 \cdot 2$ \\
Duodenum (proximal) & $588 \pm 219$ & $509 \pm 183$ \\
Duodenum (middle) & $739 \pm 186$ & $650 \pm 115$ \\
Duodenum (distal) & $321 \pm 131$ & $82 \pm 19$ \\
Jejunum (proximal) & $227 \pm 87$ & $478 \pm 176$ \\
Jejunum (middle) & $106 \pm 26$ & $327 \pm 87$ \\
Jejunum (distal) & $7 \cdot 7 \pm 2 \cdot 1$ & $16 \cdot 1 \pm 6 \cdot 0$ \\
Ileum (proximal) & $27 \pm 11$ & $<0 \cdot 2$ \\
lleum (middle) & $14 \pm 6$ & $<0 \cdot 2$ \\
Ileum (distal) & $6 \cdot 1 \pm 2 \cdot 7$ & $<0 \cdot 2$ \\
\hline
\end{tabular}

Table 1 Regional concentration of cholecystokinin in the mucosa of the gastrointestinal tract of pigs (pmol/g wet weight)

The antiserum used was specific for the sequence 25 to 30 of CCK 33 . The values are the mean \pm SEM for 4 experiments. partly in the nerves in the ileum and wholly in the 을 nerves in the colon (Larsson and Rehfeld, 1979).

THE CENTRAL NERVOUS SYSTEM

The concentration of specific CCK immuno- $\frac{\bar{\sigma}}{\bar{\omega}}$ reactivity in the porcine brain is shown in Table $2 . \overline{\mathrm{\Phi}}$ The neocortical regions contain the largest amounts of CCK, located in the neurones (Larsson and ${ }^{\infty}$ Rehfeld, 1979). The pituitary is devoid of CCK $\overrightarrow{0}$ though it does contain substantial amounts of the $\vec{\overrightarrow{ }}$ related hormone gastrin (Rehfeld, 1978d). The $\mathrm{o}_{\sigma}^{\omega}$ latter, however, is not present in any of the regions of the brain which contain abundant CCK.

MOLECULAR HETEROGENEITY OF CCK IN TISSUE Extracts of gut (Fig. 2) and brain tissue (Fig. 3) సু contain a number of different molecular forms of o CCK. The $\mathrm{NH}_{2}$ terminal composition of the largest molecular form is unknown. It is striking that octapeptide and tetrapeptide predominate in both $\stackrel{0}{5}$ intestinal and cerebral tissue. Rubin and Engel in 1973 showed that CCK 8 is significantly more potent ${ }^{\gtrless}$ than CCK 33. Chromatographic studies (Fig. 2 and $\vec{\theta}$ 3) indicate that the small molecular forms of $\mathrm{CC} \&$ are probably the more abundant hormonal forms.

\begin{tabular}{|c|c|c|}
\hline Region & Gastrin & Cholecystokinin \\
\hline Pallium & Telencephalon & \\
\hline Frontal lobe & $<0 \cdot 1$ & $556.8 \pm 103.0$ \\
\hline Parietal lobe & $<0 \cdot 1$ & $1159 \cdot 0 \pm 184 \cdot 7$ \\
\hline Temporal lobe & $<0 \cdot 1$ & $1749 \cdot 6 \pm 209 \cdot 5$ \\
\hline Occipital lobe & $<0 \cdot 1$ & $660 \cdot 3 \pm 78 \cdot 1$ \\
\hline \multicolumn{3}{|l|}{ Rhinencephalon } \\
\hline O!factory bulb & $<0.1$ & $271.4 \pm 48.6$ \\
\hline Olfactory area & $<0 \cdot 1$ & $766 \cdot 3 \pm 110 \cdot 2$ \\
\hline Hippocampus & $<0 \cdot 1$ & $136.9 \pm 26.3$ \\
\hline \multicolumn{3}{|l|}{ Corpus striatum } \\
\hline Caudate nucleus & $<0 \cdot 1$ & $375 \cdot 3 \pm$ \\
\hline Lentiform nucleus & $<0 \cdot 1$ & $234 \cdot 0 \pm 38 \cdot 8$ \\
\hline Thalamus & Diencephalon & \\
\hline Anterior thalamus & $<0 \cdot 1$ & $24 \cdot 5 \pm$ \\
\hline Posterior thalamus & $<0 \cdot 1$ & $31 \cdot 0 \pm$ \\
\hline Geniculate bodies & $<0 \cdot 1$ & $11 \cdot 4 \pm$ \\
\hline \multicolumn{3}{|l|}{ Epithalamus } \\
\hline Pineal body & $<0 \cdot 1$ & $<0 \cdot 1$ \\
\hline Thalamic habenulae & $<0 \cdot 1$ & $<0.1$ \\
\hline \multicolumn{3}{|l|}{ Hypothalamus } \\
\hline Mammilary bodies & $<0 \cdot 1$ & $36 \cdot 2 \pm 10 \cdot 7$ \\
\hline Posterior pituitary & $31 \cdot 6 \pm 3 \cdot 1$ & $<0 \cdot 1$ \\
\hline Hypothalamus & $\begin{array}{c}0 \cdot 2 \pm 0 \cdot 1 \\
\text { Mesencephalon }\end{array}$ & $110 \cdot 0 \pm 26 \cdot 1$ \\
\hline Superior colliculus & $<0 \cdot 1$ & $15 \cdot 7 \pm 3 \cdot 9$ \\
\hline Inferior colliculus & $<0 \cdot 1$ & $52 \cdot 3 \pm 10 \cdot 2$ \\
\hline Substantia nigra & $\begin{array}{l}<0 \cdot 1 \\
\text { Metencephalon }\end{array}$ & $46 \cdot 5 \pm 11 \cdot 7$ \\
\hline Cerebellum & $<0 \cdot 1$ & $<0 \cdot 1$ \\
\hline Pons & $\begin{array}{l}<0 \cdot 1 \\
\text { Myelencephalon }\end{array}$ & $2.4 \pm 0.9$ \\
\hline Medulla oblongata & $<0.1$ & $15 \cdot 0 \pm 2 \cdot 7$ \\
\hline
\end{tabular}

Table 2 Immunoreactive gastrin and cholecystokinin in the central nervous system of pigs (pmol/g wet weight)

Hormone concentrations were determined by specific radioimmuno 0 assay. Values are mean \pm SEM for 4 experiments. 


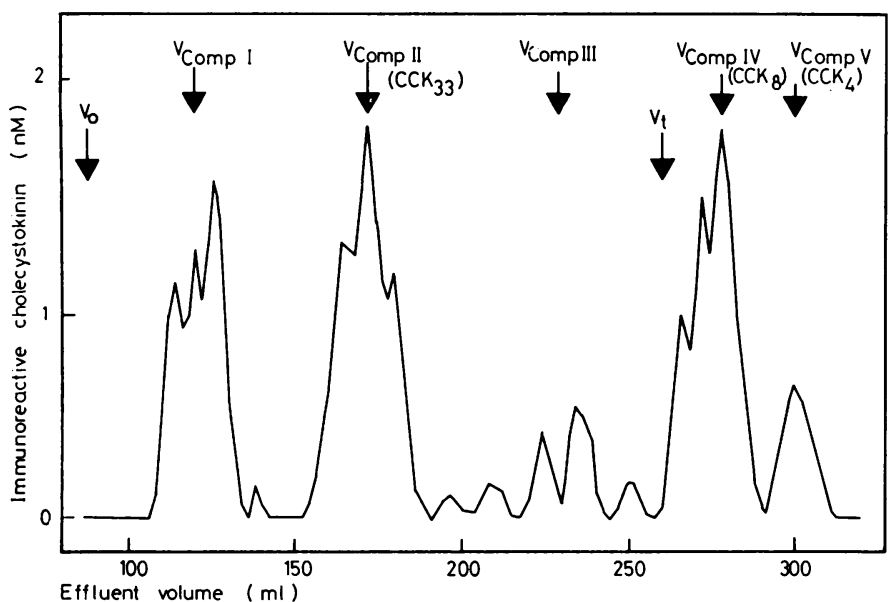

Fig. 2 Molecular heterogeneity of cholecystokinin in an acid extract of porcine jejunum as determined by gel chromatography (Sephadex G50 columns) monitored by $\mathrm{COOH}$ terminal specific antisera. The peak of CCK 4 represents $1 / 30$ th of the molar concentration.

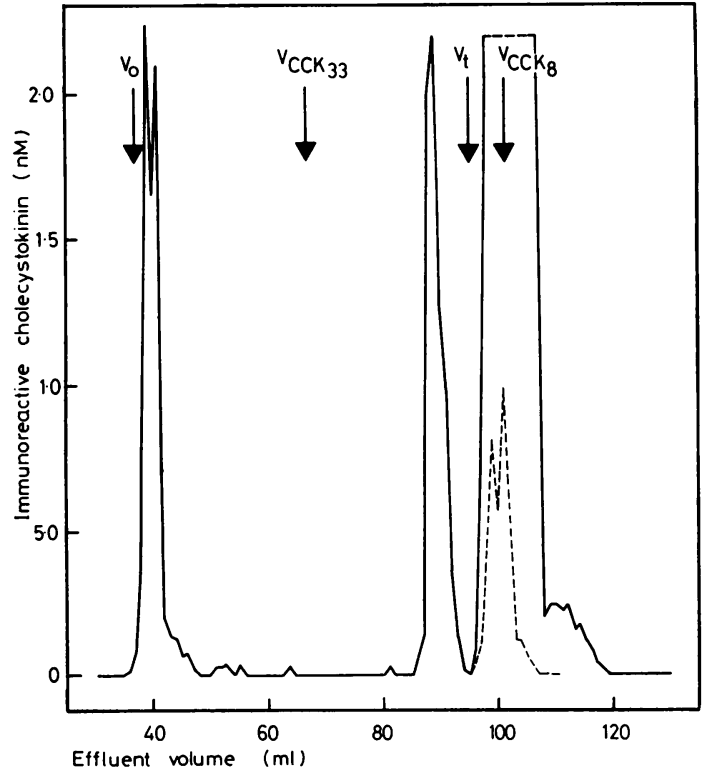

Fig. 3 Molecular heterogeneity of cholecystokinin in a neutral extract of guinea pig cerebral cortex as determined by gel chromatography (Sephadex G50 coiumns) monitored by $\mathrm{COOH}$ terminal specific antisera. The broken line represents measurements made on $a 1$ in 6 dilution.

This may imply that CCK 33 and larger forms are biosynthetic precursors.

The measurement of cholecystokinin in biological fluids

CEREBROSPINAL FLUID

Unextracted cerebrospinal fluid (CSF) from normal human subjects was found to contain only molecular forms corresponding to CCK 8 and the two tetrapeptide fragments from this (Rehfeld and KruseLarsen, 1978). This observation supports the contention that the small molecular forms of CCK are the principal biologically active forms. The CCK in CSF probably originates from brain tissues, although it is not yet known whether CCK passes the blood-brain barrier. There is no correlation between serum and CSF concentrations of gastrin over a large range of serum concentrations (Rehfeld and Kruse-Larsen, 1978).

\section{PLASMA}

As previously stated, we have not yet succeeded in measuring CCK in plasma. There are four major problems. (1) The concentration of CCK in plasma is probably very low. Measurements with crossreacting gastrin antisera suggest a concentration of a few pmol/l or less. (2) Plasma proteins interfere greatly with our CCK radioimmunoassays. (3) There is a possibility that CCK binds to plasma proteins. (4) Plasma enzymes degrade CCK.

Thus, although a great deal of progress has been made, we still have to show changes in blood concentration as evidence of the release of the hormone in response to physiological stimuli. Because of the difficulties of measuring the concentration in plasma we recently measured CCK in the perfusate from isolated porcine duodenum (unpublished observations). We showed the release of small molecular forms in response to the introduction of hydrochloric acid and fat into the duodenum, but the concentrations were very low. The measurement of CCK in blood thus requires assays specific for the $\mathrm{NH}_{2}$ terminal sequence of CCK 8 of hitherto unattained sensitivity. 


\section{References}

Bernard, C. (1856). Leçons de Physiologie Experimental, Vol. 2, p. 429. Baillière, Paris.

Bodanszky, M., Chaturvedi, N., Hudson, D., and Itoh, M. (1972). Cholecystokinin-pancreozymin. I. The synthesis of peptides corresponding to the Nterminal sequence. Journal of Organic Chemistry, 37, 2303-2307.

Dockray, G. J. (1977). Immunoreactive component resembling cholecystokinin octapeptide in intestine. Nature (London), 270, 359-361.

Fleig, C. (1903). Réflexe de l'acide sur la sécrétion biliare. Comptes Rendus des Séances de la Société de Biologie et de ses Filiales, 55, 353-355.

Fleig, C. (1904). Du mode d'action des excitants chimiques des glands digestives. Archives Internationales de Physiologie et de Biochimie, 1, 286-347.

Grossman, M. I. (1970a). Gastrin, cholecystokinin and secretin act on one receptor. Lancet, 1, 1088-1089.

Grossman, M. I. (1970b). Proposal: use the term cholecystokinin in place of cholecystokinin-pancreozymin. Gastroenterology, 58, 128.

Hunter, W. M. (1971). Radioimmunoassay Methods, p. 76, ed K. E. Kirkham and W. M. Hunter. Churchill Livingstone, Edinburgh.

Ivy, A. C., Kloster, G., Drewyer, G. E., and Lueth, M. C. (1930). Preparation of secretin concentrate. American Journal of Physiology, 95, 35-39.

Ivy, A. C., and Oldberg, E. (1928). Hormone mechanism for gall-bladder contraction and evacuation. American Journal of Physiology, 86, 599-613.

Jorpes, J. E., and Mutt, V. (1966). Cholecystokinin and pancreozymin, one single hormone? Acta Physiologica Scandinavica, 66, 196-202.

Larsson, L. I., and Rehfeld, J. F. (1977a). Evidence for a common evolutionary origin of gastrin and cholecystokinin. Nature (London), 269, 335-338.

Larsson, L. I., and Rehfeld, J. F. (1977b). Characterization of antral gastrin cells with region-specific antisera. Journal of Histochemistry and Cytochemistry, 25, 1317-1321.

Larsson, L. I., and Rehfeld ,J. F. (1979). Localization and molecular heterogeneity of cholecystokinin in the central and peripheral nervous system. Brain Research, 165, 201-218.

Muller, J. E., Straus, E., and Yalow, R. S. (1977). Cholecystokinin and its COOH-terminal octapeptide in the pig brain. Proceedings of the National Academy of Sciences of the USA, 74, 3035-3037.

Mutt, V. (1964). Behaviour of secretin, cholecystokinin and pancreozymin to oxidation with hydrogen peroxide. Acta Chemica Scandinavica, 18, 2185-2186.

Mutt, V. (1976). Further investigations on intestinal hormonal polypeptides. Clinical Endocrinology, (Suppl.), 175s-183s.

Mutt, V., and Jorpes, J. E. (1967). Isolation of aspartylঙึ? phenylalanine amide from cholecystokinin-pancreoo zymin. Biochemical and Biophysical Research Como munications, 26, 392-397.

Mutt, V., and Jorpes, J. E. (1968). Structure of porcined cholecystokinin-pancreozymin. I. Cleavage with thrombin and with trypsin. European Journal ofs Biochemistry, 6, 156-162.

Mutt, V., and Jorpes, J. E. (1971). Hormonal poly: peptides of the upper intestine (Abst.). Biochemicatu Journal, 125, 57P-58P.

Ondetti, M. A., Pluššec, J., Sabo, E. F., Sheehan, J. T 융 and Williams, N. (1970). Synthesis of cholecystokininis pancreozymin. I. The c-terminal dodecapeptide. Journal of the American Chemical Society, 92, 195-199:-

Rehfeld, J. F. (1978a). Problems in the technology of radioimmunoassays for gut hormones. In Gut Hor-O mones, pp. 112-119, ed S. R. Bloom. Churchilf Livingstone, Edinburgh.

Rehfeld, J. F. (1978b). Immunochemical studies on cholecystokinin. 1. Development of sequence-specific? radioimmunoassays for porcine triacontatriapeptide cholecystokinin. Journal of Biological Chemistry, 253, 4016-4021.

Rehfeld, J. F. (1978c). Immunochemical studies gno cholecystokinin. 2. Distribution and molecular heterogeneity in the central nervous system and smollo intestine of man and hog. Journal of Biologitur Chemistry, 253, 4022-4030.

Rehfeld, J. F. (1978d). Localisation of gastrins to neuro-0 and adenohypophysis. Nature (London), 271, 771-773. ฉ

Rehfeld, J. F., and Kruse-Larsen, C. (1978). Gastrin and $\Rightarrow$ cholecystokinin in human cerebrospinal fluids. Immunochemical determination of concentrations and molecular heterogeneity. Brain Research, 155, 19-26.

Rubin, B., and Engel, S. L. (1973). Some biologicalcharacteristics of cholecystokinin (CCK-PZ) ando synthetic analogues. In Frontiers in Gastrointestina? Hormone Research (Nobel Symposia, 16), p. 41, 3 ed S. Andersson. Almqvist and Wiksell, Stockholm.

Sandblom, P. (1933). The function of the human gall-o bladder studied in connection with blood transfusions and after stomach operations. Acta Radiologica, 14,, 249-258.

Uvnäs-Wallensten, K., Rehfeld, J. F., Larsson, L. I., and Uvnäs, B. (1977). Heptadecapeptide gastrin in the vagal nerve. Proceedings of the National Academyos of Sciences of the USA, 74, 5707-5710.

Wertheimer, E. (1903). De l'action des acides et duN chloral sur la sécrétion biliaire (d'après les expéri- $\omega$ ences de M. Ch. Dubois). Comptes Rendus des? Séances de la Société de Biologie et de ses Filiales, 55,0 286. 